NOTICE

OG 791.

PORTIONS OF THIS REPORT ARE ILLEGIBLE. II DISL- 35105 has been reproduced from the best available
copy to permit the bradest possible avail-

DE84 016994 atility.

\title{
HEAVY IONS AT STEAMBOAT: SUMMARY OF PARALLEL SESSIONS
}

\author{
Thomas W. Ludlam \\ Brookhaven National Laboratory, Upton, New York 11973 \\ Presented at the CONFERENCE on the INTERSECTIONS between \\ PARTICLE and NUCLEAR PHYSICS
}

Seamboat Springs, Colorado

May 23-30, 1984

\section{DISCLAIMER}

\begin{abstract}
This report was prepared as an account of work sponsored by an agency of the United States Government. Neither the United States Government nor any agency thereof, nor any of their employees, makes any warranty. express or implied, or assumes any legal liability or responsibility for the accuracy, completeness, or usefulness of any information, apparatus, product, $c$. process disclosed, or represents that its use would not infringe privately owned rights. Ref :rence herein to any specific commercial product, process, or service by trade name, tradem .rk, manufacturer, or otherwise does not necessarily constitute or imply its endorsement, re ummendation, or favoring by the United States Government or any agency thereof. The views and opinions of authors expressed herein do not necessarily state or reflect those if the United States Government or any agency thereof
\end{abstract}

The submitced manuscript has been authored under contract DE-ACU2-76CH00016 with the U.S. Department of Energy. Accordingly, the U.S. Government retains a nonexclusive, royalty-free license to publish or reproduce the published form of this contribution, or allow others to do so, for J.S. Government purposes. 


\title{
HEAVY IONS AT STEAMBOAT: SUMMAKY OF PARALLEL SESSIONS
}

\author{
T. Ludlam \\ - Brookhaven National Laboratory, Upton, New York 11973
}

Earlier in this meeting we were reminded by John Kogut of the dictum, attributed to Sigmund Freud, that to understand the ordinary it is often useful to study the extremes.

It is certainly true that the interest in heavy ions at the intersection between particle and nuclear physics is motivated by the opportunity for an entirely new approach to the understanding of fundamental interactions by studying extreme states of nuclear matcer. At this conference we have seen important new results on some of the central issues:

- How well can we predict the landscape of the extremes-that is, the phase structure of $O r D$ and nuclear matter?

- Can we explore it with heavy ion collisions?

- Can we recognize the appearance of new terrain?

Our present understanding of the behavior of nuclear matter under extreme conditi is is illustrated in Fig. 1 (G. Baym, Ref. 1). Here temperature is plotted vs. net baryon density for an extended volume of nuclear matter in thermal equilibrium. Normal nuclear watter appears at the point $\rho_{\mathrm{nm}}$ at zero temperature, and this is the neighborhood explored by low energy nuclear physics. Along the density axis one finds a liquid/gas phase and, at high densities, a possible pion-condensed phase. The shaded band shows the regions of temperature and density at which QCD predicts a phase transition of nuclear matter from a system of nucleons to a deconfined plasma of quarks and gluons. The critical parameters for such a transition have been caiculated from bag models. ${ }^{2}$ of more interest recently has been the success of lattice gauge simulations in which the critical behavior and limiting phases are derived from a fundamental descrj-ption of finite temperature OCD.

Fig. 2 shows the result of a lattice gauge calculation ${ }^{3}$ indicating twc coupled first-order phase transitions. At zero temperature quarks are confined in hadrons ( $h=0$ corresponds to infinite selfenergy for a free quark) and chiral symmetry is spontaneously broken (a non-zero quark-antiquark mass term, $\langle\bar{Y} Y\rangle$, in the QCD Lagrangian). At a critical temperature $T_{c}=200 \mathrm{MeV}$ both quantities undergo a discontinuous traasition corresponding to the deconfinement of quarks and gluons and the restoration of chiral symmetry. Calculations of this sort, including that shown in Fig. 2, have been incomplete in that the effects of vacuum polarization ( $i . e .$, the "sea quarks") are ignored. Theorists have questioned the relevance of these so-called "quenched approximation" calculations to QCD, and it has been a widely held view that the sharp phase transition seen in Fig. 2 would be washed out in a more realistic calculation. 
New results ${ }^{4}$ shown by John Kogut at this coaference indicate that this may not be the case after all. This work, in which light dynamical fermions are included in the computer simulation through a microcanonical ensemble, finds no qualitative difference in the phase structure from that of earlier calculations. One still finds a sharp transition, with deconfinement and chiral symmetry restoration above a critical temperature whose value is similar to that obtaind in the quenched approximations. This work is something of a computational tour de force ( $=300$ Cray hours), and the authors stress that some of the results from the simulation are not get theoreticaliy understood. Nonetheless, this is an important advance and should be a watershed for much work to follow.

The question of how the thermodynamic conditions for such a phase transition can be achieved in ultra-relativistic collisions of nuclear beams has been the topic of several presentations at this meeting. These have included elegant and ambitious designs for new accelerator facilities dedicated to this physics. ${ }^{5}$ The crucial question of nuclear stopping power, which governs the energy deposition in such collisions is taken up in detail by Alfred Goldhaber.

From a new generation of cosmic ray experiments, we are getting a glimpse of the extraordinary final states which are the signature of high energy density in very energetic collisions of heavy nuclei. New results from the Japanese American Collaborative Emulsion Experiment (JACEE) ${ }^{7}$ were presenter here by $Y$. Takahashi. This experiment flies emulsion stacks in balloons above the atmosphere. Here incident "beans" of nuceli with energies ranging up to hundreds of $\mathrm{TeV}$ and masses extending roughly up to iron are available for study. The detector, consisting of many layers of plastic and emulsion interl red with plates of dense metallic absorber, measures the charge of t...: incident projectile nucleus and provides a nearly complete measurement of charged and neutral secondaries. The experiwent has recorded $=150$ erents in which the total incident energy exceeds $10 \mathrm{TeV}$. The projectile nuclei are typically Si, A, Ca, Fe. of these events, seven have $>500$ charged secr-laries, and two have charged particle miltiplicity greater than 10:U. All of the high multiplicity events have calculated energy density (determined from the angular density of produced particles near $90^{\circ}$ in the $\mathrm{cm}$ ) in excess of $2 \mathrm{GeV} / \mathrm{fm}^{3}$ : well into the range for which the deconfined plasma phase is predicted. Since the experiment makes no selection on impact parameter, it is reasonable to conclude that the high multipliuity events correspond to head-on collisions of small impact parameter, and that among such events, given the observed yield of high multiplicity final states, events of high energy density are fairly typical.

If these high energy densities can ultimately be realized in laboratory experiments, can we recognize new phenomena in the data thus obtained? Can we, for instance, establish the existence of a phase transition? Some of the results presented here are illuminating in this regard.

Ail experiment performed recent $l y^{8}$ with internal gas jet targets in the Fermilab proton accelerator has reported evidence for the 
liquid-gas phase transition indicated at low densities in Fig. 1. In a talk given here, "David Boal discussed the theortical expectations. The phase transition is expected to occur with a critical temperature in the range $16-20 \mathrm{MeV}$, at a density of about one-half that of normal nuclear matter. These conditions should be accessible in $\mathrm{p}$-nucleus collisions, and an expected signature is a yield of fragment masses $\left(A_{F}\right)$ proportional to

$$
A_{F}-t
$$

where the exponent, $t$, should lie in the range $2-3$.

The experimental data, shown in Fig. 3, have just this

behavior. The measured value for the exponent is $2.6 \pm 2$. This would appear to be a compelling indication for the existence of the predicted phase transition. However a phase transition j.s not required by the data, and the calculated curve shown in Fig. 3 includes no phase transition. One does not have an iron-clad case. This fllustzates the difficulty of inferring thermodynamic properties of a complex configuration of nuclear interactions from single-particle inclusive measurements. One needs to constrain more completely the properties of individual events which go into such a distribution, and this in general implies multiparticle corinlation measurements.

In a similar vein, both Tanaka:0 and Zajcl' have described results from very high energy collisions of alpha particle beams at the ISR in which apparent ruclear enhancements in the amount of energy carried by particles eritted near $90^{\circ}$ in the c.m. can be well described by a model in which proton-proton sfectra are convoluted to similate multiple collisions in the alpha-alpha case.

The power of detailed specification of multiparticle final states is appreciated in two recent Bevalac experiments, $12,: 3$ where the properties of hydrodynamic flow and the compression of nuclear matter are observed in samples of events for which a large fraction of the final-state four-momentum space are determined from the directions and energies of final-state particles as indicated in Fig. 4. In collisions of heavy nuclear beams at $400 \mathrm{MeV} / \mathrm{amu}$ (the heaviest studied to date are $\mathrm{Nb}$ beams on a Nb target) an elii soidal shape is found with an axis which is not in general aligned along the beam direction. This corresponds to a fluid-like behavior of the interacting ruclear matter which cannot be the result of a simple cascade mechanisw. Comparing the observed particle production with a simple model, the authors estimate that typically $10 \%$ of the kinetic energy of collision goes into compression of nuclear matter. In an analysis which is conceptually similar, though technically very different, the GSI-LBL streamer chamber group is able to extract an equation of state relating the compressional energy to the nuclear matter density during the collision. 3 The results of this analysis are shown in in Fig. 5.

The analysis of interference effects in two-particle correlations among identical particles continues to be refined as a tool for studying extent in space and time of the particle-emitting source in nuclear collisions. At the Bevalac, systematic studies 
are under way ${ }^{1+}, 15$ to carry out these measurements with very massive beams and targets, while at the ISk such measufements have While each of these results is an advance in its own right, the overall picture presented here is equally important for the prospects of future experiments with very high energy beams of heavy ions. It seems that one can very selectively delineate the thermodynamic environment in specific event samples, and even in single events. By such selection experiments can focus on events--possibly representing a very tiny fraction of the total cross section-in which the conditions are most favorable for the formation of new types of natter at high temperatures and densities. In these events, ther, one can look for the more direct signals carried by photons, lepton pairs, jets, etc. As these direct signals were not extensively discussed at this conference, the reader is referred to the proceedings of the recent Quark Matter 183 Conference, and the Workshof n Detectors for Relativistic Nuclear Collisions, held at LBL in March, 1984, for which proceedings will be available soon.

\section{REFERENCES}

1. G. Baym, Proc. Third Int. Conf. or Ultra-Relativistic Nucleus-Nucleus Collisions (T. Ludlam and H.D. Wegner, Eds.), Nucl. Phys. A418, $433\left(198^{\prime}\right)$.

2. See, for examp!e, H. Satz, Phys. Rev. 88, 349 (1982).

3. J. Kogut et a1., Phys. Rev. Lett. 50, $\overline{393}$ (1983).

4. J. Polonyi, H. Wyld, J. Kogut, J. Shigemetsu, D. Sinclair, "Finite Temperature Phase Transitions in SU(3) Lattice Gauge Theory with Dynamical Light Fermions," these proceedings.

5. Presentations by G. Young, L. Schroeder and N.P. Samios at this conference.

6. A.S. Goldhaber, "In Search of the Quark-Gluon Plasma," and "Nuclear Stopping Power," talks presented at this conference.

7. Y. Takaheshi, presentations at this meeting; see also W.V. Jones, Ref. 1, P. 39.

8. A.S. Hirsch, "Fragment Production and the Liquid-Gas Phase Transition," Talk presented at this conference. See also A.S. Hirsch et al., Ref. 1, p. 267.

9. D. Boal, "Low energy phase transitions in Nuclear Matter," Talk presented at this conference.

10. M. Tanaka, "Study of the Neutral Energy Spectrum in the Centra: Region of ad Interactions at $\gamma_{S}=12+\mathrm{GeV}$ at the CERN ISR," Talk presented at this conference.

11. W. Zajc, "Parcicle Production in aa Collisions at the CERN ISR," Talk presented at this conference.

12. H.G. Ritter, "Collective Flow and High Density Nuclear Matter," talk presented at this conference; see also H.A. Gustafsson et al., Phys. Kev. Lett. 52, 1590 (1984).

13. A. Sandoval, "ivuclear Matter Equation of State," talk presented at this conference; see also J.W. Harris and R. Stock, LBL-17054 ( 1984 ). 
14. S.Y. Fung, "Source Size Measurements in High Energy Nucleus-Nucleus Collisions and First Results on Uranium in Streamer Chamber at Vevalac," talk presented at this conference.

15. K. Crowe, "in Correlation Measurements in Fe + Fe Collisions," talk presented at this conference.

16. 'T. Akesson et a1., Phys. Lett. 129B, 269 (1983).

FIGURE CAPTIONS

Eig. 1. Phase diagram of nuclear matter (Ref. 1).

Fig. 2. Predicted phase changes for SU(3) gauge theory on $4 \times 8^{3}$ lattice, in the quenched approximation (Ref. 3).

Fig. 3. Fragment mass yields for proton-krypton collisions (Ref. 9).

Fig. 4. Illustrating the momentum-flow analysis of Ref. 12. The evant shape is obtained by calculating a sphericity tensor.

Fig. 5. Cordressional energy, W, vs. nuclear density (Ref. 13). The resillt is obtained by comparing the configuration of finai-state pions, observed in a streamer chamber, with model calculations. 


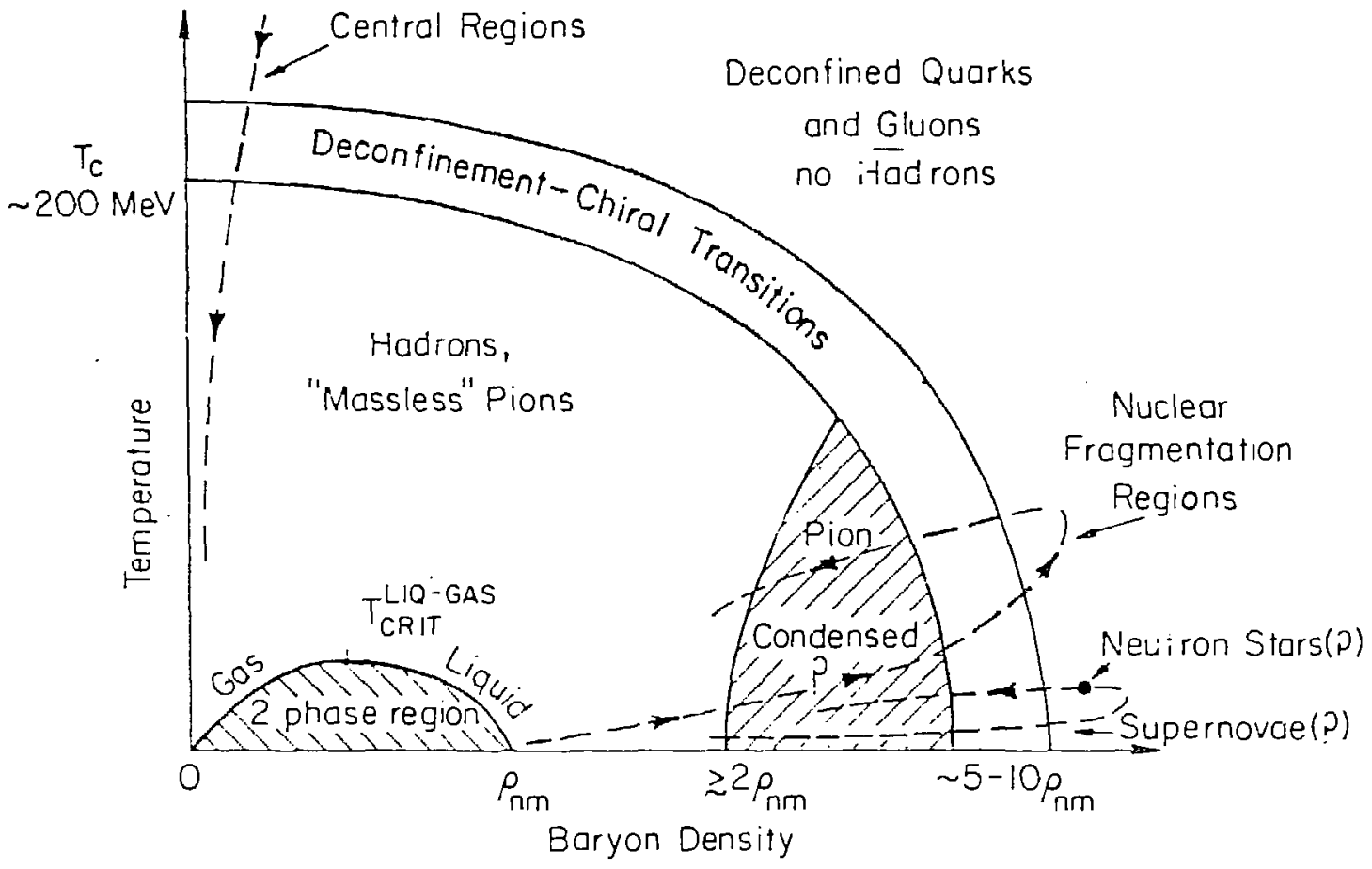

Fig. 1 


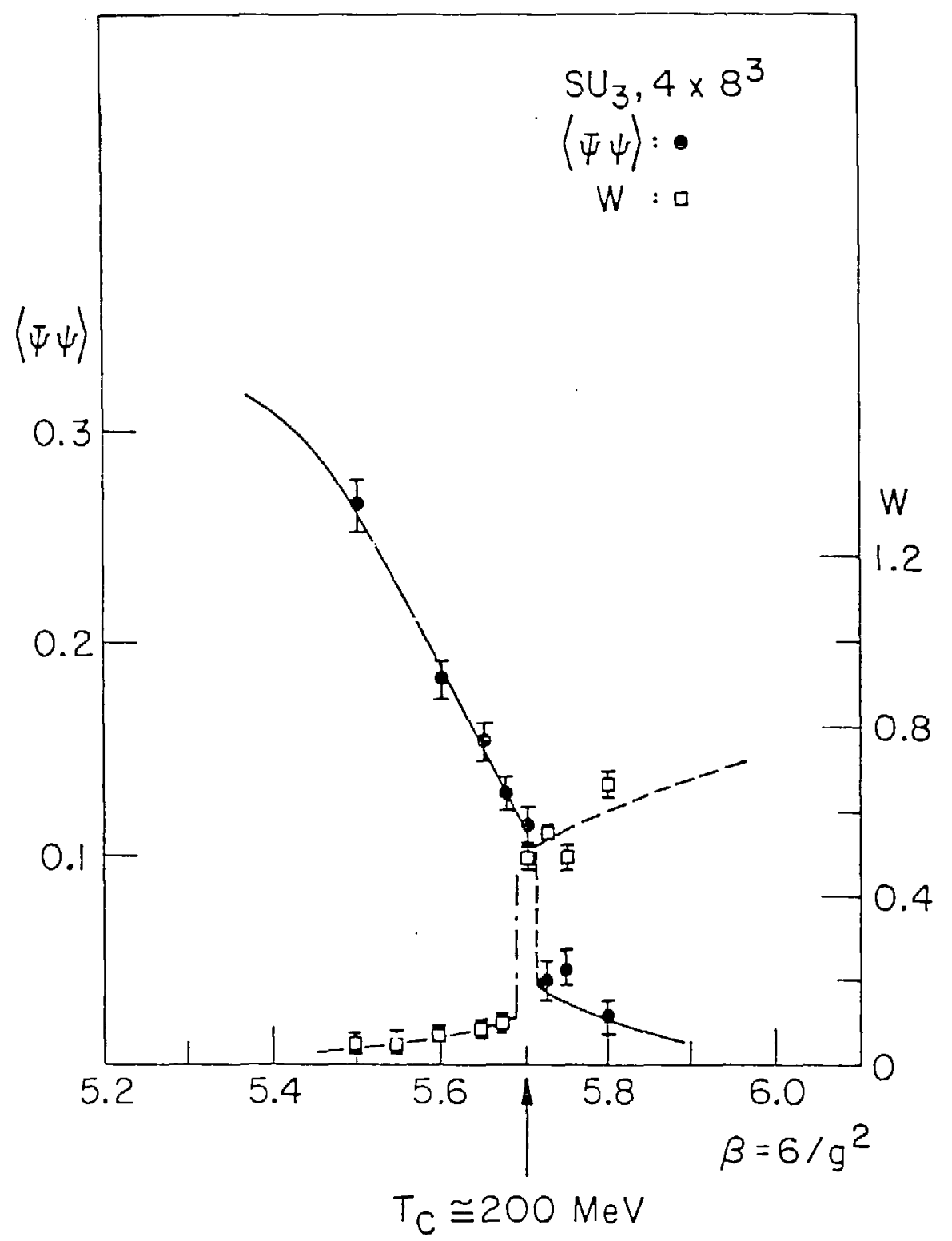

Fig. 2 


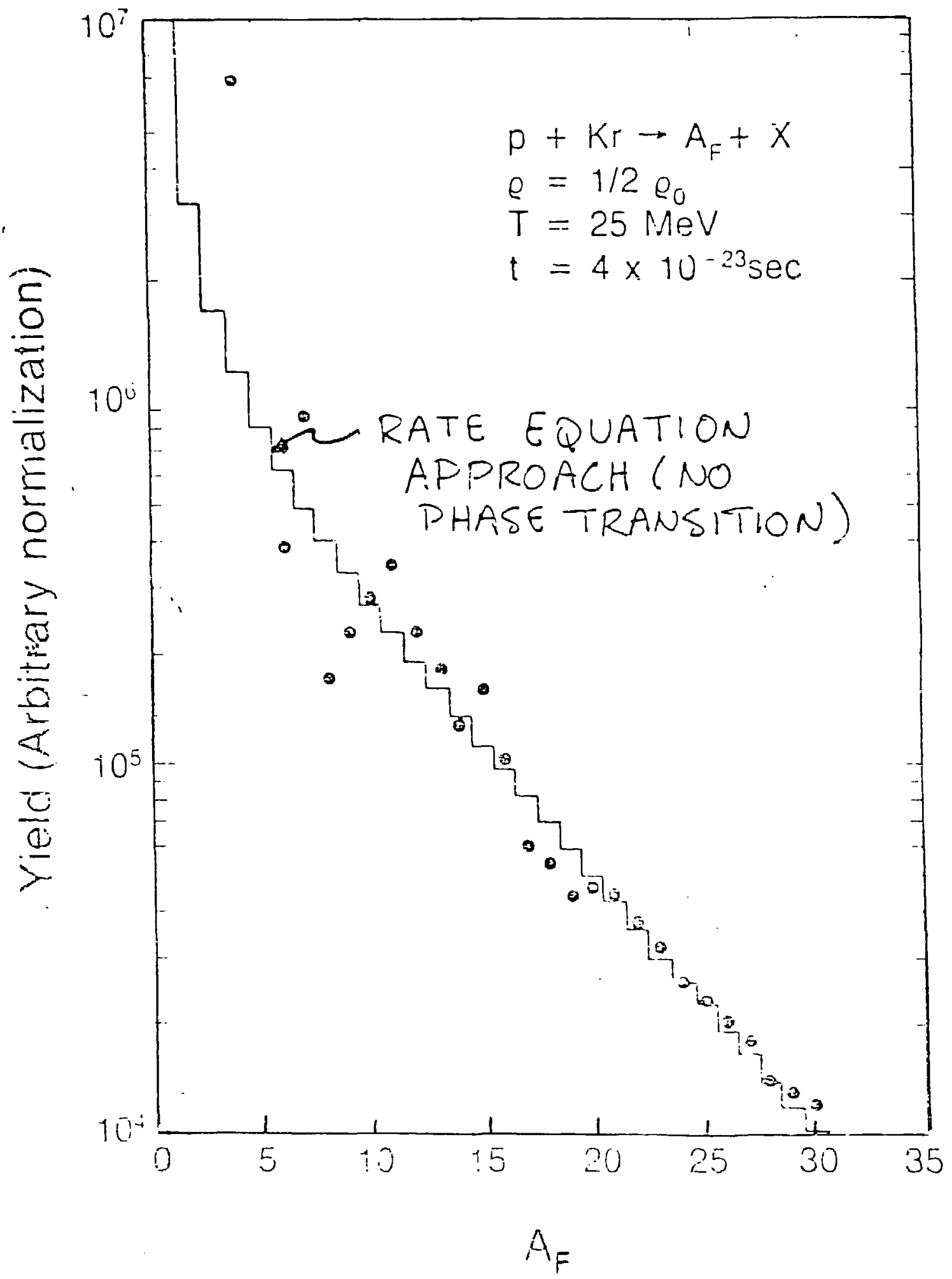

$x 92838-3127$

Fig. 3 


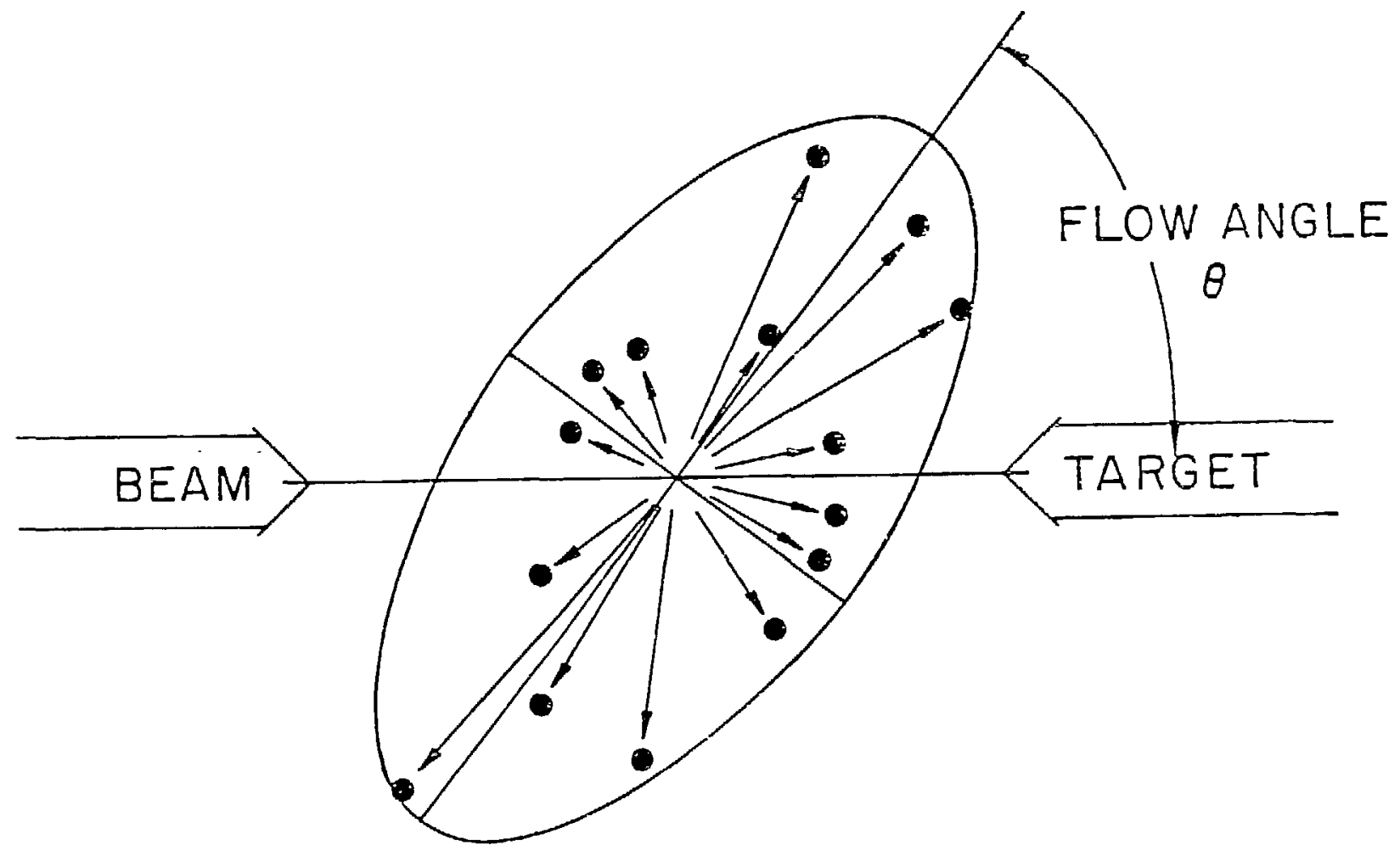

Fig. 4 


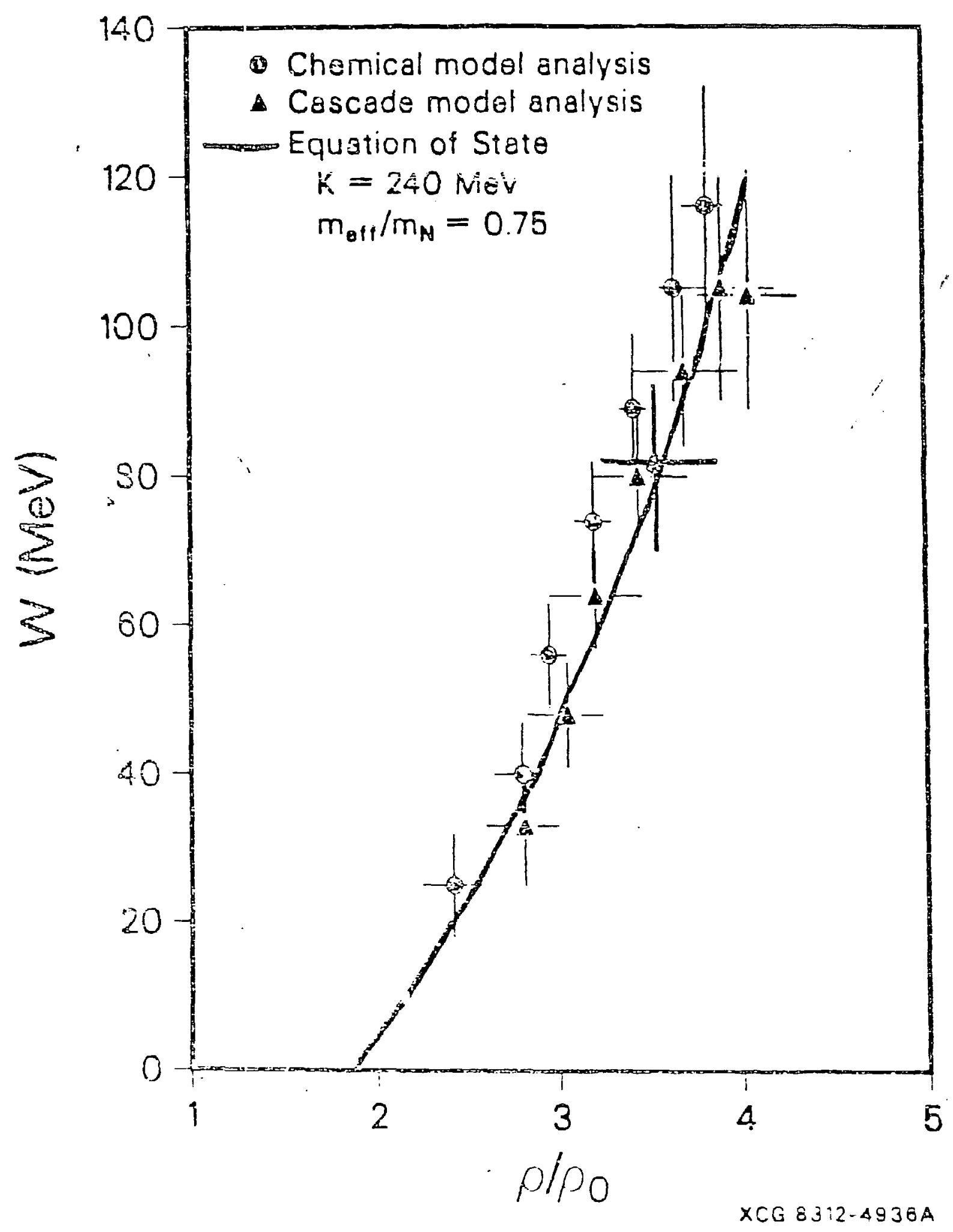

Fig. 5 


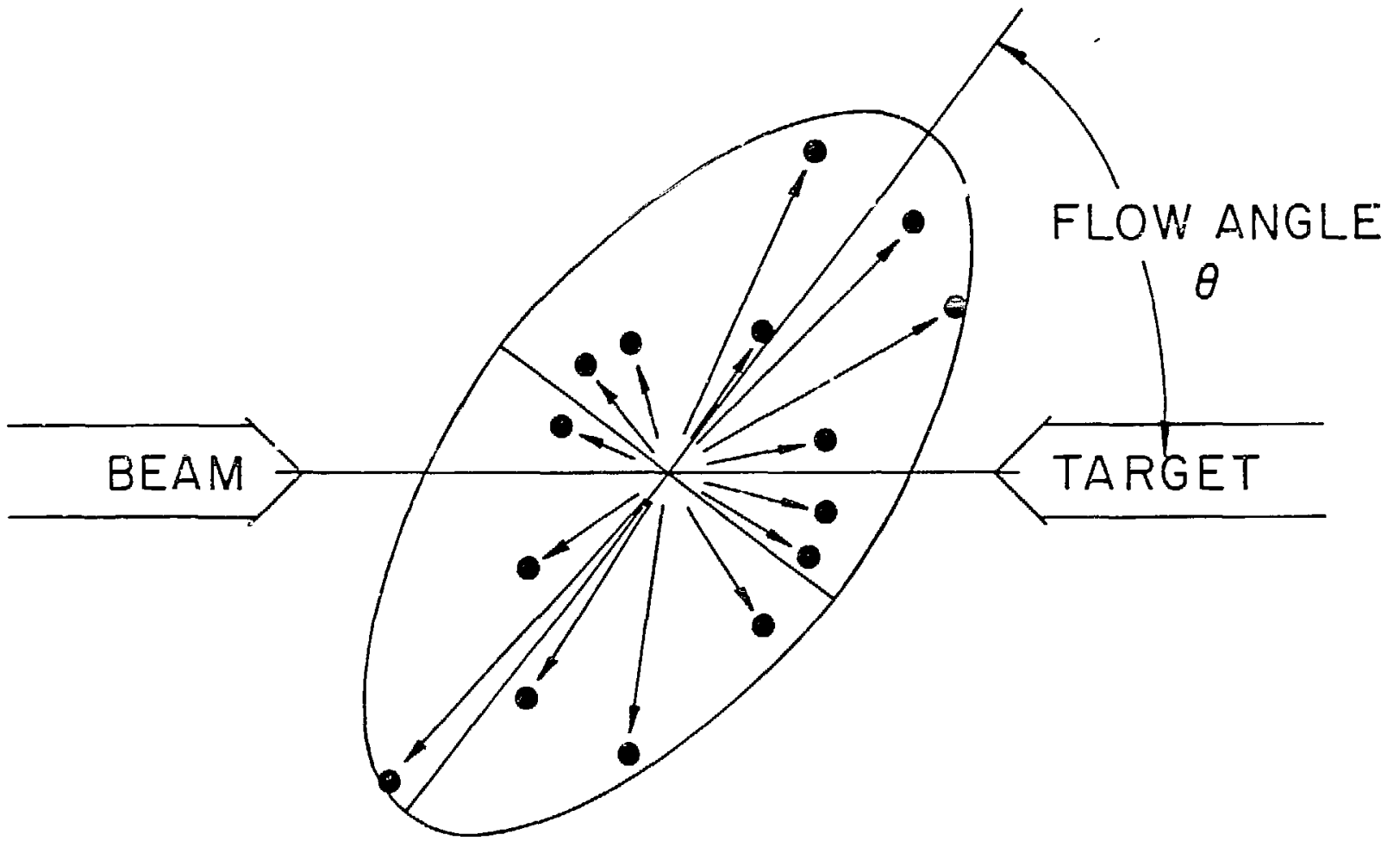

Fig. S 